\title{
A NOVEL METHOD FOR THE DESIGN OF SWITCHING SURFACES FOR DISCRETIZED MIMO NONLINEAR SYSTEMS
}

\author{
José Darío LUIS-DELGADO ${ }^{a, *}$, BAsil Mohammed AL-HADITHI ${ }^{a, b}$, AgUstín JimÉNEZ $^{a}$
}

\author{
${ }^{a}$ Intelligent Control Group, Centre for Automation and Robotics UPM-CSIC \\ Polytechnic University of Madrid, Calle José Gutierréz Abascal, 2, CP: 28006 Madrid, Spain \\ e-mail: jd.luis@alumnos.upm.es \\ ${ }^{b}$ Department of Electrical, Electronics, Control Engineering and Applied Physics \\ Higher Technical School of Industrial Design and Engineering \\ Polytechnic University of Madrid, C/Ronda de Valencia, 3, 28012-Madrid, Spain
}

\begin{abstract}
Designing variable structure control with sliding mode (VSC-SM) control schemes needs a switching function or a sliding surface which guarantees the global stability of the closed-loop system. Despite the fact that a wide range of design approaches has been proposed for solving this mathematical problem, the number of proposed methodologies for nonlinear systems is not very extensive, especially for discrete time nonlinear MIMO systems, and most of them require some coordinate system transformation. Therefore, it is not an easy task to find a design scheme that can be applied to discrete time nonlinear MIMO systems. The proposed methodology introduces a mathematical tool: a switching surface equation for a class of MIMO nonlinear systems through an explicit equation without any coordinate transformation. This equation makes use of an implicit linearizing process via the Taylor expansion that allows the use of linear procedures for the design of switching surfaces and the forward Euler method to obtain a discrete time dynamics representation. An illustrative example is included to show the advantages of the proposed design methodology.
\end{abstract}

Keywords: discrete sliding mode, variable structure control, nonlinear control, optimal control, MIMO systems.

\section{Introduction}

The procedure for designing VSC-SM consists of two steps. Firstly, designing a switching function or a sliding surface that guarantees the global stability of the closed loop system, and secondly, specifying a control law that assures the existence of a stable sliding mode. The first step is related to the closed-loop sliding phase or steady state controlled system dynamics, and the second one to the reaching phase or transient state system dynamics. Therefore, the performance of the steady state of the controlled system depends on the switching function parameters. The earliest works on VSC-SM covered this problem, mainly for continuous linear systems (Utkin, 1977; Utkin and Yang, 1978; Hung et al., 1993; DeCarlo et al., 1988; Gao and Hung, 1993). In the mid-1980s, some detailed studies on discrete time variable structure control (DVSC) appeared and, consequently, an increasing number of research works engaged in analyzing and

* Corresponding author specifying discrete time sliding surfaces were carried out (Furuta, 1990; Gao et al., 1995).

For sliding surface design in the context of linear systems, the research lines are based on classical linear system design methods such as arbitrary eigenvalues assignment (Ackermann and Utkin, 1994; Utkin et al., 1999; Perruquetti and Barbot, 2002; Draženović et al., 2013) or optimal control approaches (Tapia et al., 2014; Kim et al., 2000; Choi, 2003; Edwards and Spurgeon, 2003; Lin et al., 2013). On the other hand, for nonlinear systems, a reduced number of results have been reported. Nevertheless, the problem has been analyzed since the mid-1980s (DeCarlo et al., 1988; Sira-Ramírez, 1986; 1991; Spurgeon and Davies, 1993; Su et al., 1996; Ghaffari and Yazdanpanah, 2008; Zhang et al., 2010; Rui and Dong-wei, 2011; Nadzinski et al., 2012).

The most general methodology consists in obtaining a nonlinear canonical dynamics representation (reduced canonical form, normal canonical form, Brunovsky's 
canonical form, etc.), and then the switching surface is calculated in the new state space coordinates. Representations in normal and Brunovsky's canonical forms are most commonly used (Sira-Ramírez, 1991), but cannot be applied to nonminimum phase plants.

On the other hand, if a coordinate transformation is used, then the switching surface specification is made in the new coordinate system, and therefore an inverse transformation is needed in order to obtain knowledge about the dynamics in the original one. Furthermore, for high order plants, the complexity of these approaches increases considerably and finding the solution becomes a difficult task. In addition, existing research works are based on the ideal sliding mode (SM) dynamics, i.e., they do not take into consideration the nonideal behaviour of the SM inherent to DVSC (Gao et al., 1995; Sira-Ramírez, 1991; Milosavljević, 1985; Potts and Yu, 1991; Furuta and Pan, 1995). These nonideal dynamics, known as the quasi-sliding or pseudo-sliding mode, consist of undesirable oscillatory vector state trajectories around the switching surface. These drawbacks (the lack of design methodologies, the complexity of the few existent ones and the need for considering quasi-sliding mode dynamics) motivate our research work, whose main objective is to find an easy way for specifying a stable sliding manifold for discrete time nonlinear multi-input-multi-ouput (MIMO) systems considering the nonideal SM dynamics in the design procedure.

The proposed design methodology is based on a new versatile switching surface equation applied to obtaining an MIMO discrete time nonlinear system with the following main features: (i) the switching surface equation has the same structure regardless of the system nonlinearities dynamics, (ii) the complexity of applying the methodology does not vary with the order of the plant, (iii) some parameters of the proposed equation allow obtaining different steady state performances according to various design criteria such as optimal control methods or eigenvalue assignment approaches, (iv) the proposed methodology does not need any coordinate transformation, and (v) a design parameter is considered for the adjustment of the nonideal sliding mode system dynamics.

All these features make the methodology a useful tool. Indeed, the designer can vary some parameters of the equation to obtain several switching surfaces and, consequently, a comparison of the resultant sliding mode dynamics with other techniques can be made.

Finally, it is important to stress that, for achieving the switching surface scheme, the forward Euler time stepping method (or the explicit Euler method) is used to obtain a discrete time difference equation approximation and the Taylor expansion is applied to linearize the system. In the work is introduced of Draženović et al. (2013), a decoupled solution for designing switching surfaces applied to linear continuous and discrete time systems without any transformation using a state feedback gain matrix which can be chosen using eigenvalues or optimal methodologies. The main advantage of the proposed methodology, compared with this approach, is that it includes a design parameter which allows the adjustment of the sliding mode dynamics of the controlled system.

An overview of the general sliding hyperplane designing problem related to MIMO nonlinear systems is presented in Section 2. The remainder of the paper is organized as follows: the proposed design method is developed in Section 3, an illustrative design example is detailed in Section 4, and conclusions and future proposals are presented in Section 5.

Throughout this paper, the following notation is used: $f: \mathbb{R}^{m} \rightarrow \mathbb{R}^{n}$ stands for a function that maps from the $m$-dimensional vector space $\mathbb{R}^{m}$ to the $n$-dimensional vector space $\mathbb{R}^{n} ;\|\cdot\|$ denotes the magnitude of a scalar or the standard Euclidean norm of a vector; $|\cdot|$ indicates the absolute value of a scalar; $\operatorname{sign}(\cdot)$ stands for the sign of a real scalar and $\operatorname{det}(\cdot)$ for the determinant of a square matrix; $I_{m}$ means the $m \times m$ identity matrix; $\mathcal{N}(\cdot)$ represents the null space of a matrix; $\mathcal{R}(\cdot)$ denotes the range of a matrix and $\Lambda(\cdot)$ means the set of eigenvalues of a square matrix. Finally, $\mathcal{P}(\cdot)$ denotes a generic system or process.

\section{Preliminaries}

VSC and DVSC are control schemes where the input laws $\mathbf{u}: \mathbb{R}^{m} \rightarrow \mathbb{R}^{n}$ are generally noncontinuous functions that depend on the value of some switching function $\mathbf{s}: \mathbb{R}^{n} \rightarrow$ $\mathbb{R}^{m}$ such that the state of the systems, denoted as $\mathbf{x} \in$ $\mathbf{X} \subset \mathbb{R}^{n}$, is steered from some initial state $\mathbf{x}(0) \in \mathbf{X}$ to a subspace region $\mathcal{S}=\{\mathbf{x} \in \mathbf{X}: \mathbf{s}(\mathbf{x})=0\}$. Therefore, the system state remains indefinitely on $\mathcal{S}$, i.e., the state slides on the surface. Consequently, $\mathcal{S}$ is known as a switching surface and, once the state arrives and lies on the switching surface, the system is said to be in SM.

A particular feature of VSC (see Fig. 1) is that these controllers are built with two main parts: the first one is a multivariable function known as a switching surface (the box labelled as $\mathbf{s}(\mathbf{x}(k))$ in the figure) and a bank of control law blocks or a big multivariable function that makes use of the output of the switching surface in order to obtain the desired control action $\mathbf{u}(k)$ to the plant. As previously mentioned, the aim of this work is to give a new methodology for the design switching surfaces applied to MIMO nonlinear systems. The general nonlinear sliding surface problem can be stated as follows.

Problem 1. Consider a nonlinear discrete time system 
whose dynamics are defined as

$$
\begin{aligned}
\mathbf{x}(k+1) & =\mathbf{f}(\mathbf{x}(k), \mathbf{u}(k), k), \\
\mathbf{y}(k) & =\mathbf{h}(\mathbf{x}(k), \mathbf{u}(k), k),
\end{aligned}
$$

where $\mathbf{x} \in \mathbf{X} \subset \mathbb{R}^{n}, \mathbf{u} \in \mathbf{U} \subset \mathbb{R}^{m}$ and $\mathbf{y} \in \mathbf{U} \subset \mathbb{R}^{p}$ are the state, input and output vectors, respectively, and $f: \mathbf{X} \times \mathbf{U} \rightarrow \mathbf{X}$ and $h: \mathbf{X} \times \mathbf{U} \rightarrow \mathbf{Y}$ are supposed to be analytical functions. Then, given a control law $u(k)$ such that a sliding mode on the surface $\mathcal{S}$ is ensured:

$$
\mathcal{S}=\{\mathbf{x} \in \mathbf{X} \mid \mathbf{s}(\mathbf{x}(k))=0\},
$$

the switching function $\mathbf{s}: \mathbf{X} \rightarrow \mathbb{R}^{m}$ must be specified to make the closed-loop system asymptotically stable.

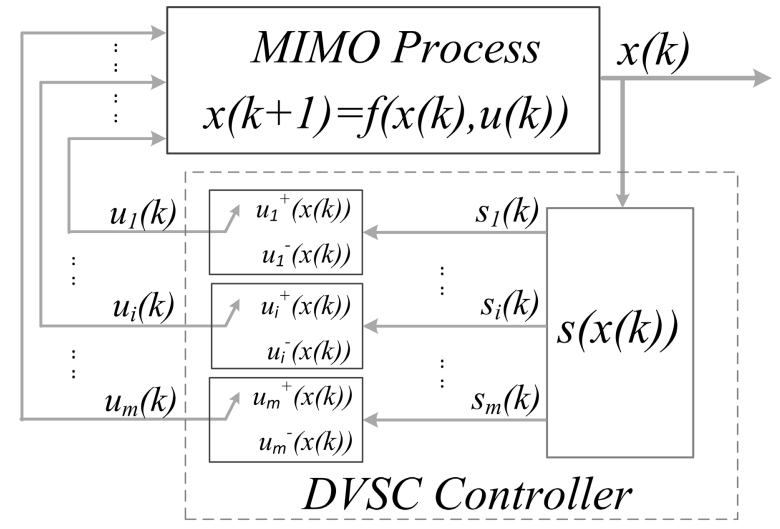

Fig. 1. General DVSC scheme.

It has been mentioned that the number of research works related to the design of switching surfaces applied to nonlinear systems is considerably smaller than for linear ones. One of the earliest works related to nonlinear sliding surface design was presented by Sira-Ramírez (1986), who proposed to obtain a linearized equivalent representation via state feedback, where a linear sliding surface is designed in Brunovsky's canonical form. Other approaches make use of linear approximations as in the work of Camacho and Smith (2000), where nonlinear systems are approximately modelled via first order plus dead time (FOPDT). In another work, Sira-Ramírez (1991) proposed the use of the normal canonical representation for the design of DVSC for nonlinear systems, but without any indication or guideline about how the switching sliding surfaces are chosen. DeCarlo et al. (1988) presented a method for designing SM surfaces using regular canonical representations (Johansson and Nunes, 1998), and Spurgeon and Davies (1993) made use of a noncanonical transformation. Su et al. $(1996$; 1994) set forth a Lyapunov approach for sliding surface design. More recently, Ghaffari and Yazdanpanah (2008) proposed a method for computing nonlinear stable sliding mode surfaces, and Rui and Dong-wei (2011) introduced an algorithm for designing optimal sliding surface for nonlinear discrete time systems using the nonlinear two point boundary problem (TPBV) approach, while Bartoszewicz and Leśniewski (2014) made use of an optimal approach for designing a sliding hyperplane. Finally, some approaches intended to apply DVSC with an SM to discrete time nonlinear systems based on $T-S$ fuzzy models use linear methods such as LMI for the design of switching surfaces (Zhang et al., 2010; Nadzinski et al., 2012).

Before presenting the design of sliding surfaces applied to nonlinear systems, let us find the ideal SM dynamics. Consider a nonlinear discrete time system whose dynamics are defined in (1). Then the equivalent control law $u_{e q u}(k)$ can be found so that

$$
\mathbf{s}(k+1)=\mathbf{s}(k)=0,
$$

where

$$
\mathbf{s}(k+1)=\mathbf{s}\left(\mathbf{f}\left(\mathbf{x}(k), \mathbf{u}_{e q u}(k), k\right)\right) .
$$

This equation yields the ideal sliding mode described as

$$
\begin{aligned}
\mathbf{x}(k+1) & =\mathbf{f}\left(\mathbf{x}(k), \mathbf{u}_{e q u}(k), k\right), \quad k \in \mathbb{Z}^{+}, \\
\mathbf{s}(\mathbf{x}(k)) & =0, \quad \forall k .
\end{aligned}
$$

For a linear switching surface $\mathbf{s}(k)=\mathbf{C x}(k)$ and from (1), we obtain

$$
\mathbf{C f}\left(\mathbf{x}(k), \mathbf{u}_{e q u}(k), k\right)=0 .
$$

Therefore

$$
\mathbf{u}_{e q u}(k)=\mathbf{f}^{-1}(\mathbf{x}(k), \mathbf{u}(k), k),
$$

which implies that $\mathbf{u}_{e q u}$ can be unequivocally found if $\mathbf{f}$ is single-valued or regular.

In the case of affine systems, i.e., those whose dynamics exhibit a linear behaviour in relation to the inputs and a nonlinear one with respect to the states, (1) can be written as

$$
\mathbf{x}(k+1)=\mathbf{f}(\mathbf{x}(k), k)+\mathbf{\Gamma} \mathbf{u}(k),
$$

where $\boldsymbol{\Gamma}: \mathbf{X} \rightarrow \mathbf{X} \times \mathbf{U}$. Thus, the ideal sliding surface dynamic are defined as

$$
\mathbf{s}(k)=\mathbf{C f}(\mathbf{x}(k), k)+\mathbf{C} \mathbf{u}(k),
$$

and the equivalent control law is given by

$$
\mathbf{u}_{e q u}(k)=-(\mathbf{C} \boldsymbol{\Gamma})^{-1} \mathbf{C f}(\mathbf{x}(k), k) .
$$

Therefore, the closed loop ideal SM is described as

$$
\mathbf{x}(k+1)=\left[\mathbf{I}_{n}-\boldsymbol{\Gamma}(\mathbf{C} \boldsymbol{\Gamma})^{-1} \mathbf{C}\right] \mathbf{f}(x(k), k) .
$$


In general, the methods for designing nonlinear surfaces are based on obtaining a suitable canonical representation in order to find an easy mathematical model that could help in the design process (Hung et al., 1993; DeCarlo et al., 1988; Gao and Hung, 1993; Sira-Ramírez 1986; 1991; Spurgeon and Davies, 1993; Su et al., 1994). The following section presents a new scheme for designing sliding switching surfaces applied to MIMO discrete time nonlinear systems, which simplifies the design tasks and gives a high level of accuracy for an arbitrary location of eigenvalues or optimal SM dynamics, depending on the desirable design criteria.

\section{Proposed sliding hyperplane design: The main results}

A novel sliding surface equation is introduced in the next theorem.

Theorem 1. Suppose that we are given the following:

1. a MIMO nonlinear continuous time system $\mathcal{P}(\cdot)$ with dynamics described as

$$
\dot{\mathbf{x}}(t)=\mathbf{f}(\mathbf{x}(t), \mathbf{u}(t)),
$$

where $\mathbf{x} \in \mathbf{X} \subset \mathbb{R}^{n}$ is the state vector and $\mathbf{f}$ : $\mathbb{R}^{n+m} \rightarrow \mathbb{R}^{n}$ defined as

$$
\mathbf{f}(x)=\left[\begin{array}{llll}
f_{1}(x, u) & f_{2}(x, u) & \cdots & f_{n}(x, u)
\end{array}\right]_{(13)}^{T},
$$

where every $f_{i}: \mathbb{R}^{n+m} \rightarrow \mathbb{R}$ is a smooth nonlinear function;

2. a state feedback gain matrix $\mathbf{K} \in \mathbb{R}^{m \times n}$ such that the following system dynamics are asymptotically stable:

$$
\mathbf{x}(k+1)=[\hat{\mathbf{\Phi}}-\hat{\boldsymbol{\Gamma}} \mathbf{K}] \mathbf{x}(k),
$$

where $\hat{\boldsymbol{\Phi}}$ and $\hat{\boldsymbol{\Gamma}}$ are defined as

$$
\begin{aligned}
& \hat{\mathbf{\Phi}}=\mathbf{I}_{n}+T \mathbf{J}_{A}, \\
& \hat{\boldsymbol{\Gamma}}=T \mathbf{J}_{B},
\end{aligned}
$$

$\mathbf{I}_{n}$ being the $n$-th order identity matrix, $T$ sample time and $\mathbf{J}_{A}$ and $\mathbf{J}_{B}$ the Jacobian matrix of $\mathbf{f}(x)$ with respect to $\mathbf{x}(t)$ and $\mathbf{u}(t)$, respectively, such that

$$
\begin{aligned}
& \mathbf{J}_{A}(x)=\left.\frac{\partial f}{\partial x}\right|_{x=x_{e}, u=u_{e}}, \\
& \mathbf{J}_{B}(x)=\left.\frac{\partial f}{\partial u}\right|_{x=x_{e}, u=u e},
\end{aligned}
$$

where $\mathbf{x}_{e} \in \mathbf{X}$ is an equilibrium state.
If $\mathcal{P}(\cdot)$ is controlled via DVSC, then the switching function $\mathbf{s}(k)=\mathbf{C x}(k)$ makes the discrete time ideal sliding mode of the controlled system asymptotically stable around $\mathbf{x}_{e} \in \mathbf{X}$ if $\mathbf{C}$ is estimated as

$$
\mathbf{C}=\mathbf{E K}\left[\mathbf{I}_{n}+\mathbf{T} \mathbf{J}_{A}(x)\right]^{-1},
$$

where $\mathbf{E} \in \mathbb{R}^{m \times m}$ is an arbitrary nonsingular design matrix.

Proof. Let us obtain a linearized system through the first-order term of the Taylor expansion of $\mathbf{f}(\mathbf{x}(t))$ around an equilibrium point $\mathbf{x}_{e}$ (see Section A.1 in Appendix), i.e., the system dynamics given in (12) are approximated around an equilibrium state $\mathbf{x}_{e}$ as follows:

$$
\dot{\mathbf{x}}(t) \approx \mathbf{A} \mathbf{x}(t)+\mathbf{B u}(t)
$$

where

$$
\begin{aligned}
& \mathbf{A}=\mathbf{J}_{A}(x)=\left.\frac{\partial \mathbf{f}}{\partial \mathbf{x}}\right|_{x=x_{e}, u=u_{e}}, \\
& \mathbf{B}=\mathbf{J}_{B}(x)=\left.\frac{\partial \mathbf{f}}{\partial \mathbf{u}}\right|_{x=x_{e}, u=u_{e}},
\end{aligned}
$$

Then, for time discretization, let us use the Euler method (see Section A.2 in Appendix). Hence, a discrete time system dynamics representation of (17) is equal to

$$
\begin{aligned}
\mathbf{x}(k+1) & =\left[\mathbf{I}_{n}+T \mathbf{A}\right] \mathbf{x}(k)+T \mathbf{B u}(k) \\
& =\hat{\boldsymbol{\Phi}} x(k)+\hat{\boldsymbol{\Gamma}} \mathbf{u}(k)
\end{aligned}
$$

Owing to the condition stated in (14) for the next Lyapunov function, we have

$$
V(k)=\mathbf{x}^{T}(k) \mathbf{P} \mathbf{x}(k),
$$

and the following linear Lyapunov equation holds:

$$
[\hat{\mathbf{\Phi}}-\hat{\boldsymbol{\Gamma}} \mathbf{K}]^{T} \mathbf{P}[\hat{\boldsymbol{\Phi}}-\hat{\boldsymbol{\Gamma}} \mathbf{K}]-\mathbf{P}=-\mathbf{Q}
$$

$\mathbf{P} \in \mathbb{R}^{n \times n}$ being a symmetric positive definite matrix and $\mathbf{Q} \in \mathbb{R}^{n \times n}$ a symmetric positive semi-definite matrix.

On the other hand, from (11), and on account of the switching surface $\mathbf{s}(k)=\mathbf{C x}(k)$, the ideal SM of the system given in 19) is as follows:

$$
\begin{aligned}
\mathbf{x}_{S M}(k+1) & =\left[\hat{\boldsymbol{\Phi}}-\hat{\boldsymbol{\Gamma}}(\mathbf{C} \hat{\boldsymbol{\Gamma}})^{-1} \mathbf{C} \hat{\boldsymbol{\Phi}}\right] \mathbf{x}(k) \\
& =\hat{\boldsymbol{\Phi}}_{e q u} \mathbf{x}(k) .
\end{aligned}
$$

Taking account of the SM dynamics specified in (22) and applying the Lyapunov theorem to SM stability analysis, the following condition must hold:

$$
\begin{array}{r}
{\left[\hat{\boldsymbol{\Phi}}-\hat{\boldsymbol{\Gamma}}(\mathbf{C} \hat{\boldsymbol{\Gamma}})^{-1} \mathbf{C} \hat{\boldsymbol{\Phi}}\right]^{T} \mathbf{P}\left[\hat{\boldsymbol{\Phi}}-\hat{\boldsymbol{\Gamma}}(\mathbf{C} \hat{\boldsymbol{\Gamma}})^{-1} \mathbf{C} \hat{\boldsymbol{\Phi}}\right]} \\
-\mathbf{P}=-\mathbf{Q}
\end{array}
$$


Since $\mathbf{C}=\mathbf{E K} \hat{\boldsymbol{\Phi}}^{-1}$, we get

$$
\begin{array}{r}
{\left[\hat{\mathbf{\Phi}}-\hat{\boldsymbol{\Gamma}}(\mathbf{C} \hat{\boldsymbol{\Gamma}})^{-1} \mathbf{E K}\right]^{T} \mathbf{P}\left[\hat{\boldsymbol{\Phi}}-\hat{\boldsymbol{\Gamma}}(\mathbf{C} \hat{\boldsymbol{\Gamma}})^{-1} \mathbf{E K}\right]} \\
-\mathbf{P}=-\mathbf{Q} .
\end{array}
$$

Then, defining $\mathbf{E}=\mathbf{C} \hat{\boldsymbol{\Gamma}}$ without any loss of generality and substituting it into (24), we obtain

$$
[\hat{\mathbf{\Phi}}-\hat{\mathbf{\Gamma}} \mathbf{K}]^{T} \mathbf{P}[\hat{\boldsymbol{\Phi}}-\hat{\boldsymbol{\Gamma}} \mathbf{K}]-\mathbf{P}=-\mathbf{Q},
$$

which is the same equation as the one given in (21), and this completes the proof.

Theorem 1 shows that the sliding surface $\mathbf{s}(k)=$ $\mathbf{C x}(k)$, where $\mathbf{C} \in \mathbb{R}^{m \times n}$ is defined in (16), guarantees the asymptotic stability of any MIMO nonlinear system represented as in (12) and where a state feedback gain matrix $\mathbf{K}$ is needed. Hence, the problem of designing the sliding surface $\mathbf{s}(k)=\mathbf{C x}(k)$ is reduced to the specification of the matrix $\mathbf{C}$ so that the characteristic polynomial of $\hat{\boldsymbol{\Phi}}_{C L}=\hat{\boldsymbol{\Phi}}-\hat{\boldsymbol{\Gamma}} \mathbf{K}$ is strictly Schur (Åström and Wittenmark, 1997). Additionally, the gain matrix $\mathbf{K}$ can be either estimated via arbitrary eigenvalues assignment approaches or with optimal methods such as the linear quadratic regulator (LQR) or the linear quadratic Gaussian (LQG). This can be considered an advantage of the proposed methodology because designers can obtain several Ks, and then the evaluation of SM performance can obtained for every $\mathbf{K}$. For the pole placement methodology, it is worth mentioning that $m$ eigenvalues of $\hat{\boldsymbol{\Phi}}_{C L}$ must be set to zero, because the equivalent SM always has $m$ poles at the origin (Spurgeon and Pugh, 1991). The other $n-m$ eigenvalues must be set inside the unit circle.

Theorem 2. If the gain matrix $\mathbf{K} \in \mathbb{R}^{m \times n}$ stabilizes the system via eigenvalue shifting such that the roots of the characteristic polynomial of the matrix

$$
X_{C L}(\lambda)=\operatorname{det}\left[\lambda \mathbf{I}_{n}-\hat{\mathbf{\Phi}}+\hat{\boldsymbol{\Gamma}} \mathbf{K}\right]=0
$$

are located at the arbitrary eigenvalues

$$
\lambda_{d}=\{\lambda_{1}, \lambda_{2}, \cdots, \lambda_{n-m}, \underbrace{0,0, \cdots, 0}_{m}\},
$$

where $\left|\lambda_{i}\right|<1, i=1,2, \ldots, n-m$, then the surface definition $\mathbf{s}(k)=\mathbf{C x}(k)$ makes the ideal sliding mode of the controlled system asymptotically stable with eigenvalues located exactly at $\lambda_{d}$ provided that $\mathbf{C}$ is obtained using (16).

Proof. From the theorem statement, the feedback gain matrix K stabilizes the system, where the closed-loop system dynamics are given by

$$
\mathbf{x}_{C L}(k+1)=[\hat{\mathbf{\Phi}}-\hat{\mathbf{\Gamma}} \mathbf{K}] \mathbf{x}(k) .
$$

If the system is transformed to the canonical form via a similar transformation $\overline{\mathbf{x}}(k)=\mathbf{M x}(k)$, then the dynamics of the closed-loop canonical system are given by

$$
\overline{\mathbf{x}}_{C L}(k+1)=[\overline{\mathbf{\Phi}}-\overline{\mathbf{\Gamma}} \overline{\mathbf{K}}] \overline{\mathbf{x}}(k),
$$

where $\overline{\mathbf{\Phi}}=\mathbf{M} \hat{\mathbf{\Phi}} \mathbf{M}^{-1}, \overline{\boldsymbol{\Gamma}}=\mathbf{M} \hat{\boldsymbol{\Gamma}}$ and $\overline{\mathbf{K}}=\mathbf{K} \mathbf{M}^{-1}$ and $\mathbf{M}$ is given as

$$
\mathbf{M}=\left[\begin{array}{c}
\mathbf{g}_{m_{1}} \\
\mathbf{g}_{m_{1}} \hat{\mathbf{\Phi}} \\
\vdots \\
\mathbf{g}_{m_{1}} \hat{\mathbf{\Phi}}^{m_{1}-1} \\
\mathbf{g}_{m_{1}+m_{2}} \hat{\boldsymbol{\Phi}} \\
\mathbf{g}_{m_{1}+m_{2}} \\
\vdots \\
\mathbf{g}_{m_{1}+m_{2}} \hat{\boldsymbol{\Phi}}^{m_{2}-1} \\
\vdots \\
\mathbf{g}_{m_{1}+\cdots+m_{p}} \hat{\boldsymbol{\Phi}}^{m_{p}-1}
\end{array}\right]
$$

with $\mathbf{g}_{i}$ is being the $i$-th row of the rearranged controllability matrix $\overline{\mathbf{W}}_{c}$ obtained from $\mathbf{W}_{c}$ as follows:

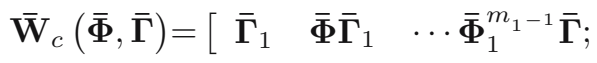

$$
\begin{aligned}
& \begin{array}{lll}
\overline{\boldsymbol{\Gamma}}_{2} & \overline{\mathbf{\Phi}} \overline{\boldsymbol{\Gamma}}_{2} & \overline{\boldsymbol{\Phi}}_{2}^{m_{2-1}} \overline{\boldsymbol{\Gamma}}
\end{array} \\
& \text {... } \\
& \begin{array}{llll}
\overline{\boldsymbol{\Gamma}}_{m_{i}} & \overline{\mathbf{\Phi}} \overline{\boldsymbol{\Gamma}}_{m_{i}} & \cdots & \left.\overline{\boldsymbol{\Phi}}_{p}^{m_{p}-1} \overline{\boldsymbol{\Gamma}}\right],
\end{array}
\end{aligned}
$$

where $\sum_{i=1}^{p} m_{i}=n$. It is known that a similar transformation is invariant to the eigenvalue location, and therefore the eigenvalues of $[\overline{\mathbf{\Phi}}-\overline{\mathbf{\Gamma}} \overline{\mathbf{K}}]$ are $\lambda_{d}$. As for the ideal SM of the system in the canonical form, we have

$$
\overline{\mathbf{x}}_{S M}(k+1)=\left[\overline{\mathbf{\Phi}}-\overline{\boldsymbol{\Gamma}}(\overline{\mathbf{C}} \overline{\boldsymbol{\Gamma}})^{-1} \overline{\mathbf{C}} \overline{\mathbf{\Phi}}\right] \hat{\mathbf{x}}(k) .
$$

Comparing (28) and (22), it is clear that if the following condition is fulfilled:

$$
\overline{\mathbf{K}}=(\overline{\mathbf{C}} \overline{\boldsymbol{\Gamma}})^{-1} \overline{\mathbf{C}} \overline{\mathbf{\Phi}}
$$

then the eigenvalues of the ideal SM matrix are located at $\lambda_{d}$. Also, let us consider the following representation for $\overline{\mathbf{C}}$ and $\bar{\Gamma}$ :

$$
\begin{aligned}
& \overline{\mathbf{C}}=\left[\begin{array}{llll}
\overline{\mathbf{c}}_{1} & \overline{\mathbf{c}}_{2} & \cdots & \overline{\mathbf{c}}_{n}
\end{array}\right], \\
& \overline{\boldsymbol{\Gamma}}=\left[\begin{array}{c}
\mathbf{0}_{m_{1}-1 \times m} \\
\mathbf{F}_{1} \\
\mathbf{0}_{m_{2}-1 \times 1} \\
\mathbf{F}_{2} \\
\vdots \\
0 \\
\mathbf{F}_{m}
\end{array}\right],
\end{aligned}
$$


where $\overline{\mathbf{c}}_{i} \in \mathbb{R}^{m}$ is the $i$-th column of $\mathbf{C} \in \mathbb{R}^{m}$. Defining the matrix $\mathbf{F} \in \mathbb{R}^{m \times m}$ as

$$
\mathbf{F}=\left[\begin{array}{r}
\mathbf{F}_{1} \\
\mathbf{F}_{2} \\
\vdots \\
\mathbf{F}_{m}
\end{array}\right]
$$

and taking account of the fact that

$$
\mathbf{F}_{i}=\left[\begin{array}{lllllll}
0 & 0 & \cdots & 1 & * & \cdots & *
\end{array}\right]
$$

we conclude that $\mathbf{F}$ is an upper triangular matrix in which all the elements on the main diagonal are ones. Therefore, $\operatorname{det}(\mathbf{F})=1$. In addition, it can be shown that

$$
\begin{aligned}
\overline{\mathbf{C}} \overline{\boldsymbol{\Gamma}} \mathbf{F}^{-1} & =\mathbf{L} \\
& =\left[\begin{array}{llll}
c_{m_{1}} & c_{m_{1}+m_{2}} & \cdots & c_{n}
\end{array}\right] .
\end{aligned}
$$

Thus, if $\mathbf{L}$ is arbitrarily specified such that $\operatorname{det}(\mathbf{L})=$ $\alpha, \alpha \neq 0$, since $\operatorname{det}\left(\mathbf{F}^{-1}\right)=1$, we get $\operatorname{det}(\overline{\mathbf{C}} \overline{\boldsymbol{\Gamma}})=\alpha \neq$ 0. Defining $\mathbf{E}=\mathbf{F L F}=\overline{\mathbf{C}} \overline{\boldsymbol{\Gamma}}$ and replacing $\overline{\mathbf{C}} \overline{\boldsymbol{\Gamma}}$ into (32), we obtain

$$
\overline{\mathbf{K}}=\mathbf{E}^{-1} \overline{\mathbf{C}} \overline{\boldsymbol{\Phi}}
$$

Therefore

$$
\overline{\mathbf{C}}=\mathbf{E} \overline{\mathbf{K}} \overline{\boldsymbol{\Phi}}^{-1}
$$

Finally, from $\mathbf{C}=\overline{\mathbf{C}} \mathbf{M}, \overline{\mathbf{K}}=\mathbf{K M}^{-1}$ and $\mathbf{M}^{-1} \overline{\boldsymbol{\Phi}}^{-1} \mathbf{M}=\hat{\boldsymbol{\Phi}}^{-1}$, it follows that

$$
\mathbf{C}=\mathbf{E K} \hat{\boldsymbol{\Phi}}^{-1}
$$

Corollary 1. Under the assumptions of Theorem 2 we have $\mathbf{E}=\mathbf{C} \hat{\boldsymbol{\Gamma}}$ when $\mathbf{K}$ fulfills (26) and (27).

From Theorem 2, the spectrum of the ideal SM dynamics, denoted as $\Lambda\left(\hat{\boldsymbol{\Phi}}_{e q u}\right)$, have $m$ zero eigenvalues and the same $n-m$ eigenvalues chosen for the stable dynamics of $\hat{\boldsymbol{\Phi}}_{C L}$, i.e., $\Lambda\left(\hat{\boldsymbol{\Phi}}_{C L}\right)=\Lambda\left(\hat{\boldsymbol{\Phi}}_{e q u}\right)$, where $\hat{\boldsymbol{\Phi}}_{\text {equ }}$ is given in (22). As shown in Theorem 2, the sliding surface definition (16) presents a simple way to configure the SM dynamics by means of an arbitrary eigenvalues assignment and it is easy to check that the eigenvalues of the resultant ideal SM system matrix are located exactly at the desired eigenvalues. What is more, the theorem can be proven easier, with the hint chosen intended to prove that the ideal sliding mode is completely specified by $\mathbf{K}$. Moreover, if the arbitrary matrix $\mathbf{E}$ is chosen such that $\mathbf{E}=\mathbf{C} \hat{\boldsymbol{\Gamma}} \neq 0$, then the SM invariance property is guaranteed (Dorling and Zinober, 1986). Since $\mathbf{C} \hat{\boldsymbol{\Gamma}} \neq 0$, we have $\mathcal{R}(\boldsymbol{\Gamma}) \cap \mathcal{N}(C)=\varnothing$, which implies that $\mathbf{E}$ does not affect the ideal SM dynamics and, therefore, they are exclusively specified by the gain matrix $\mathbf{K}$.

However, Milosavljević (1985) proved that for discrete time systems the ideal SM cannot be achieved and a quasi-or pseudo-sliding mode appears. In the quasi-sliding mode, the state vector oscillates in a region around $\mathbf{s}(k)=0$ and control laws are designed for minimizing the amplitude of oscillations. Hence, calculating the nonideal SM dynamics yields

$$
\mathbf{s}(k+1)=\mathbf{E}[\mathbf{K x}(k)+\mathbf{u}(k)] .
$$

Then it is obvious that $\mathbf{s}(k+1)$ depends on $\mathbf{E}$. Indeed, every $\mathbf{s}_{i} \in \mathbf{s}(k)$ can be adjusted through a linear combination of the components of the vector $\mathbf{u}(k)$. Consequently, E should be considered when the control laws are specified. It is important to note that if the state feedback gain matrix $\mathbf{K}$ does not fulfill the conditions given in (26) and (27), then $\mathbf{E} \neq \mathbf{C}^{-1} \hat{\boldsymbol{\Gamma}}$. However, it can be shown that a desired $\mathbf{C} \boldsymbol{\Gamma}=\mathbf{D}$ can be achieved if $\mathbf{E}$ is defined as follows:

$$
\mathbf{E}=\mathbf{D}\left(\mathbf{K} \hat{\boldsymbol{\Phi}}^{-1} \hat{\boldsymbol{\Gamma}}\right)^{-1} .
$$

In addition, some relevant features that are very helpful during the design of control laws are given in the following lemma.

Lemma 1. Consider $\mathcal{P}(\mathbf{x}, \mathbf{u})$, a linearized and dis cretized MIMO system whose dynamics are described by (19). If $\mathcal{P}(\mathbf{x}, \mathbf{u})$ is controlled using a DVSC and the switching surface is defined as in (16) such that the restrictions (26) and (27) are fulfilled, then the ideal SM equivalent control $\mathbf{u}_{e q u}(k)$ is equal to

$$
\mathbf{u}_{e q u}(k)=-\mathbf{K} \mathbf{x}(k),
$$

and the ideal SM dynamics are

$$
\begin{aligned}
\mathbf{s}(k+1) & =\mathbf{s}(k)=0, \\
\mathbf{x}_{\text {equ }}(k+1) & =\mathbf{\Phi}_{\text {equ }} \mathbf{x}(k),
\end{aligned}
$$

where

$$
\Phi_{e q u}=\boldsymbol{\Phi}-\boldsymbol{\Gamma K} .
$$

Proof. Note that the proof is immediate if we set $\mathbf{C}=\mathbf{E K} \hat{\boldsymbol{\Phi}}^{-1}$ in (10) and (11).

This lemma introduces two important features: (i) the equivalent control law is similar to the classical state feedback control law for pole placement, and (ii) the equivalent system dynamics do not depend on e and are exactly the same as for the system controlled using and state space feedback scheme.

Considering Theorem 1, the proposed design method can be described as follows:

1. Obtain the matrices $\hat{\boldsymbol{\Phi}}$ and $\hat{\boldsymbol{\Gamma}}$ using (15) and (18). The sampling time $T$ can be obtained from the system step response using the Åström criterion (Åström and Wittenmark, 1997), which establishes that the sampled time $T$ can be calculated from the system 
step response so that, during the rise time $t_{r}$, the number of samples $N_{r}$ must be between 4 and 10 , i.e.,

$$
N_{r}=\frac{t_{r}}{T}=4 \sim 10
$$

2. Find the gain matrix $\mathbf{K}$ so that $\hat{\boldsymbol{\Phi}}_{C L}=\hat{\boldsymbol{\Phi}}-\hat{\mathbf{\Gamma}} \mathbf{K}$ is strictly Schur.

3. Find any desired $\mathbf{C \Gamma}$ according to the desired performance of the pseudo-sliding system dynamics. If $\mathbf{K}$ is found using eigenvalue placement, then $\mathbf{E}=$ $\mathbf{C \Gamma}$, otherwise use the equation given in (41). If no specific $\mathbf{C \Gamma}$ definition is given, choose an arbitrary matrix E.

4. Compute $\mathbf{C}$ using (16).

5. Verify that the characteristic polynomial of $\hat{\Phi}_{e q u}$ is strictly Schur.

Once the switching surface is completely defined, a control law $u(k)$ which fulfills some reaching law restriction such as the ones given by Gao et al. (1995), Sira-Ramírez (1991), Bartoszewicz (1998) or Furuta and Pan (2002) is needed. In addition, the design procedure explained so far considers plants without uncertainties about their dynamics. It is known (Gao et al., 1995) that perturbed systems controlled via DVSC whose dynamics are given as follows:

$$
\begin{aligned}
\mathbf{x}(k+1)= & (\tilde{\boldsymbol{\Phi}}+\Delta \tilde{\boldsymbol{\Phi}}) \mathbf{x}(k) \\
& +\hat{\boldsymbol{\Gamma}} u(k)+\mathbf{d}(k)
\end{aligned}
$$

are invariant with respect to system deviations $\Delta \tilde{\boldsymbol{\Phi}}$ and external perturbations $\mathbf{d}(k)$ when the following matching conditions hold:

$$
\begin{aligned}
\Delta \tilde{\boldsymbol{\Phi}} & =\hat{\boldsymbol{\Gamma}} \tilde{\boldsymbol{\Phi}}, & & \tilde{\boldsymbol{\Phi}} \in \mathbb{R}^{m \times n}, \\
\mathbf{d}(k) & =\hat{\boldsymbol{\Gamma}} \tilde{\mathbf{d}}(k), & & \tilde{\mathbf{d}}: \mathbb{R} \rightarrow \mathbb{R}^{m},
\end{aligned}
$$

and $\operatorname{det}(\mathbf{E}) \neq 0$.

However, the control law $\mathbf{u}(k)$ must be designed such that some reaching law must be fulfilled considering these uncertainties, mostly when the matching conditions are not achieved (Furuta, 1990; Gao et al., 1995; Kim et al., 2000; Sira-Ramírez, 1991; Yu et al., 2000; Yadav and Singh, 2012; Mondal et al., 2012; Qu et al., 2014). This part of the design of DVSC controllers is not directly carried out during switching the surface design phase. Additional design problems, such as unobservable states, can be minimized using state vector estimation or predictors (see, e.g., Pai, 2008; Wang et al., 2011). In addition, it is known that for discrete time systems (Soroush and Kravaris, 1992) delays only modify the relative degree of the state space dynamics. Thus, time delay compensation can be made using some additional scheme such as the one proposed by Sira-Ramírez (1991).

\section{Example}

An industrial process used as a MIMO example is applied. It includes level control of a four-tank laboratory system (Gatzke et al., 2000). This nonlinear system consists of four-tanks $T_{1}, T_{2} T_{3}$ and $T_{4}$ with the cross-sectional area $A_{i}, i=1, \ldots, 4$, respectively, and where two pumps (system inputs) are used for transferring water from a bottom basin into four upper tanks (see Fig. 2). The output flow rate for each pump $u_{1}(t)$ and $u_{2}(t)$ can be manipulated to control the two level outputs $h_{1}(t)$ and $h_{2}(t)$, respectively. The system exhibits interacting multivariable dynamics because it was designed such that $u_{1}(t)$ and $u_{2}(t)$ affect $h_{1}(t)$ and $h_{2}(t)$. The continuous time system model dynamics are as follows:

$$
\begin{aligned}
& \dot{h}_{1}(t)=-\frac{1}{A_{1}}\left[a_{1} \sqrt{2 g h_{1}(t)}-a_{3} \sqrt{2 g h_{3}(t)}\right] \\
& +\frac{1}{A_{1}} \gamma_{1} k_{1} u_{1}(t) \text {, } \\
& \dot{h}_{2}(t)=-\frac{1}{A_{2}}\left[a_{2} \sqrt{2 g h_{2}(t)}-a_{4} \sqrt{2 g h_{4}(t)}\right] \\
& +\frac{1}{A_{2}} \gamma_{2} k_{2} u_{2}(t) \text {, } \\
& \dot{h}_{3}(t)=-\frac{1}{A_{3}}\left[a_{3} \sqrt{2 g h_{3}(t)}-\left(1-\gamma_{2}\right) k_{2} u_{2}(t)\right] \text {, } \\
& \dot{h}_{4}(t)=-\frac{1}{A_{4}}\left[a_{4} \sqrt{2 g h_{4}(t)}-\left(1-\gamma_{1}\right) k_{1} u_{1}(t)\right] \text {, } \\
& y(t)=\left[\begin{array}{llll}
1 & 1 & 0 & 0
\end{array}\right] h(t),
\end{aligned}
$$

where $d_{1}(t)$ and $d_{2}(t)$ are the deviation and perturbation signals, $g \simeq 980 \mathrm{~cm} / \mathrm{s}^{2}$ is the gravitational acceleration, $a_{i}$ and $A_{i}$ correspond to the drain and the tank area of the $i$-th tank, respectively, and where $\gamma_{1}$ and $\gamma_{2}$ denote the ratio flow in Tank 1 and Tank 4 and the ratio flow in Tank 2 to Tank 3, respectively, such that $\gamma_{1}+\gamma_{2}<1$.

The process parameters considered here are $A_{i}=$ $A=730 \mathrm{~cm}^{2}, a_{1}=a_{3}=a_{2}=a_{4}=2.3 \mathrm{~cm}^{2}$, $k_{1}=5.51, k_{2}=6.58 \mathrm{~cm}^{3} / \mathrm{s}, u_{i}^{0}=60 \%, \gamma_{1}=$ 0.333 and $\gamma_{2}=0.307$. It is easy to show that, for the inputs $u_{1}(k)=u_{2}(k)=60 \%$, the initial state $\mathbf{x}(0)=\left[\begin{array}{llll}14.192 & 11.243 & 7.212 & 4.680\end{array}\right]^{T}$ is a stationary one. Following the proposed methodology, the first step consists in finding the Jacobian matrices $\mathbf{J}_{A}, \mathbf{J}_{B}$. Hence

$$
\mathbf{J}_{A}=\left[\begin{array}{rrrr}
-\frac{1}{T_{1}} & 0 & \frac{A_{3}}{A_{1} T_{3}} & 0 \\
0 & -\frac{1}{T_{2}} & 0 & \frac{A_{4}}{A_{2} T_{4}} \\
0 & 0 & -\frac{1}{T_{3}} & 0 \\
0 & 0 & 0 & -\frac{1}{T_{4}}
\end{array}\right]
$$




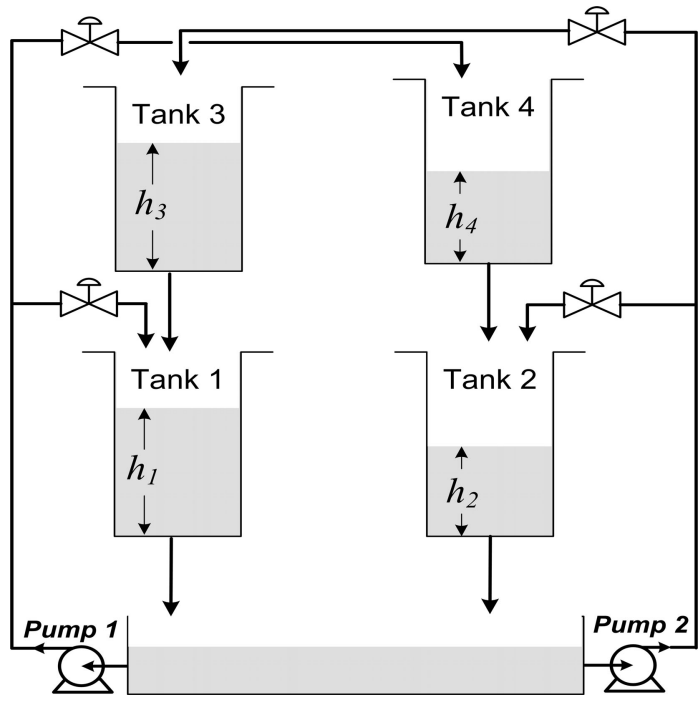

Fig. 2. Experimental four tank system scheme.

$$
\mathbf{J}_{B}=\left[\begin{array}{cc}
\frac{\gamma_{1} k_{1}}{A_{1}} & 0 \\
0 & \frac{\gamma_{2} k_{2}}{A_{3}} \\
0 & \frac{\left(1-\gamma_{2}\right) k_{2}}{A_{3}} \\
\frac{\left(1-\gamma_{1}\right) k_{1}}{A_{4}} & 0
\end{array}\right]
$$

where

$$
T_{i}=\frac{A_{i}}{a_{i}} \sqrt{\frac{2 h_{i}(0)}{g}} .
$$

The second step is to find $\hat{\boldsymbol{\Phi}}$. Defining the state vector

$$
\mathbf{x}(t)=\left[\begin{array}{llll}
h_{1}(t) & h_{2}(t) & h_{3}(t) & h_{4}(t)
\end{array}\right]^{T}
$$

and a sample time $T=0.5 \mathrm{~s}$, we have

$$
\begin{aligned}
& \mathbf{x}(k+1)=\hat{\boldsymbol{\Phi}} x(k)+\hat{\boldsymbol{\Gamma}} \mathbf{u}(k)+\mathbf{n}(k), \\
& \hat{\boldsymbol{\Phi}}=\left[\begin{array}{rrrr}
0.991 & 0 & 0.013 & 0 \\
0 & 0.990 & 0 & 0.016 \\
0 & 0 & 0.987 & 0 \\
0 & 0 & 0 & 0.984
\end{array}\right] \text {, } \\
& \hat{\boldsymbol{\Gamma}}=\left[\begin{array}{cc}
0.001 & 0 \\
0 & 0.001 \\
0 & 0.003 \\
0.0025 & 0
\end{array}\right] \text {, } \\
& \mathbf{n}(k)=\left[\begin{array}{cc}
0 & 0 \\
0 & 0 \\
0.2 & 0 \\
0 & 0.3
\end{array}\right] \mathbf{d}(k) \text {. }
\end{aligned}
$$

Since $\mathbf{x}_{r}=\mathbf{x}_{e} \neq 0$, the system dynamics must be specified in relation to the error vector $\mathbf{e}(k)$ defined as follows:

$$
\mathbf{e}(k)=\mathbf{x}_{r}-\mathbf{x}(k), \quad \mathbf{x}_{r}=\mathbf{x}_{e} .
$$

First we need to select a state vector feedback gain matrix $\mathbf{K}$ such that the system given in (52) is asymptotically stable. Choosing an optimal procedure (MATLAB function dlqr), the following gain matrix $\mathbf{K}$ is obtained:

$$
\mathbf{K}=\left[\begin{array}{rrrr}
30.967 & 39.236 & -14.700 & 82.493 \\
37.259 & 23.677 & 85.482 & -15.328
\end{array}\right] \text {, }
$$

where $\mathbf{P}$ and $\mathbf{Q}$ are arbitrarily chosen as $\mathbf{I}_{n}$ and $10^{-5} \mathbf{I}_{n}$, respectively. Then, the matrix $\mathbf{E}$ must be specified.

Choosing the following arbitrary matrix $\mathbf{E}$ :

$$
\mathbf{E}=\mathbf{E}_{1}=\left[\begin{array}{rr}
1 & 0.01 \\
0 & 1
\end{array}\right]
$$

the switching surface is computed by means of the proposed swtiching surface equation given in (16):

$$
\mathbf{C}=\left[\begin{array}{rrrr}
31.6310 & 39.8851 & -14.4379 & 83.0338 \\
37.6053 & 23.9244 & 86.1099 & -15.9645
\end{array}\right]
$$

Using (22) for calculating the system matrix $\hat{\boldsymbol{\Phi}}_{e}$ for the ideal SM, we have

$$
\hat{\mathbf{\Phi}}_{e}=\left[\begin{array}{rrrr}
0.8364 & -0.1970 & 0.0926 & -0.4184 \\
-0.1686 & 0.8837 & -0.3921 & 0.0911 \\
-0.3746 & -0.2317 & 0.1001 & 0.1848 \\
-0.3028 & -0.3897 & 0.1705 & 0.1477
\end{array}\right],
$$

and the ideal SM spectrum is

$$
\Lambda\left(\hat{\boldsymbol{\Phi}}_{S M}\right)=\{0.9889,0.9791,0,0\} .
$$

Therefore, the ideal SM is asymptotically stable. In order to illustrate the closed-loop stability, numerical simulations are performed as explained next.

Simulation. The numerical values are obtained using the computing environment MATLAB ${ }^{\circledR}$, and the controller was implemented using a control law obtained via the reaching law approach proposed by Gao et al. (1995) such that

$$
\begin{aligned}
\mathbf{u}(k)= & \mathbf{u}_{e q u}(k) \\
& -\mathbf{E}^{-1}[\mathbf{s}(k)(\mu T-1)+\varepsilon T \operatorname{sign}(\mathbf{s}(k))],
\end{aligned}
$$

where the arbitrary designing parameters $\varepsilon$ and $\mu$ must satisfy the following restriction:

$$
\Delta=\frac{\varepsilon T}{1-\mu T}
$$

where $2 \Delta$ corresponds to the width of the quasi sliding mode band (QSMB). For a QSMB $<1$ and $\mu=1, \varepsilon$ becomes 0.5 .

Tracking problem simulation. To illustrate how the system follows the reference value or set-point $\mathbf{x}_{r}(k)$, 
let us consider a scenario where $\mathbf{x}_{r}(k)$ changes from $\mathbf{x}_{r}=\mathbf{x}_{e}$ to $\mathbf{x}_{r}=\mathbf{x}_{e}(1+20 \%)$. Thus, the error vector value quickly raises up as shown in Fig. 3. Nevertheless, DVSC can drive the error vector to zero, which proves the asymptotically stability of the switching surface (see Figs. 5 and 4). Hence, the state vector signal values are driven from $\mathbf{x}(0)$ to the new reference, i.e., $\mathbf{x}(k)$ follows $\mathbf{x}_{r}(k)$ (see Fig. 5), and the surface values $\mathbf{s}(k)$, which are shown in Fig. 4, decay in a short time.

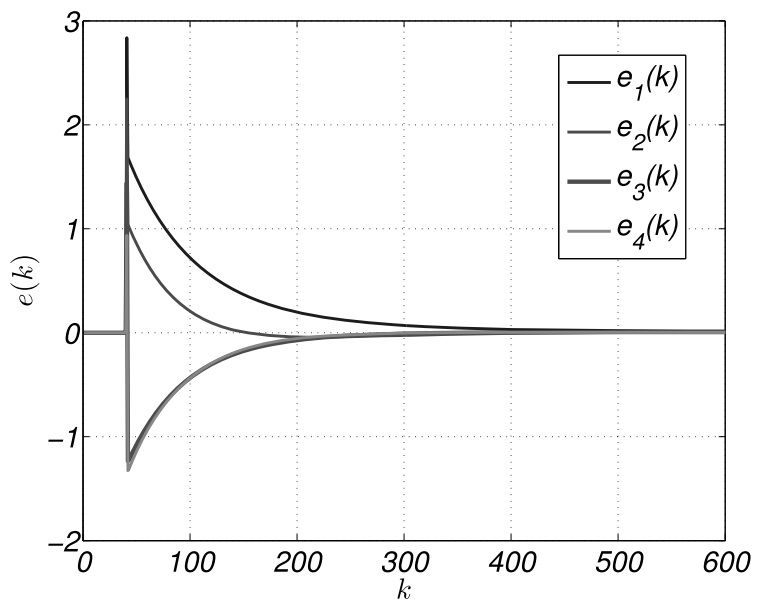

Fig. 3. Tracking error $\mathbf{e}(k)$.

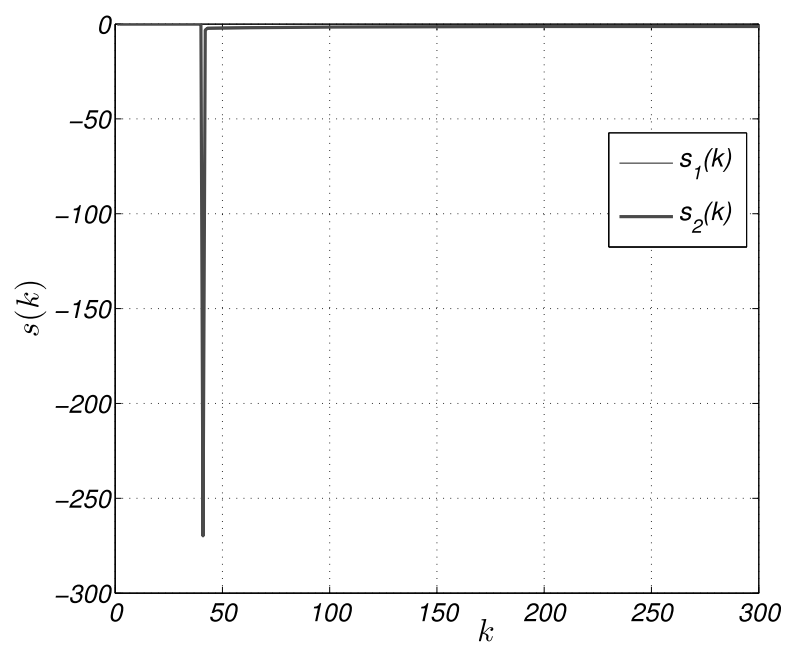

Fig. 4. Evolution of the sliding surface $\mathbf{s}(k)$.

Robustness analysis. In order to illustrate the robustness of the SM dynamics for a switching surface designed using the proposed methodology, two scenarios are presented. The first one considers an external disturbance which increases the level of Tank 3 by $0.5 \mathrm{~cm}$ for $5 \mathrm{~s}$. In Fig. 6 it can be seen that the state vector value is perturbed

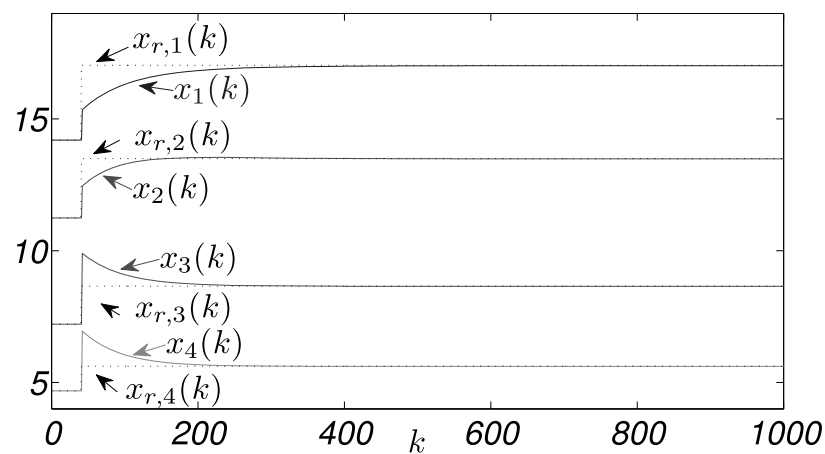

Fig. 5. Evolution of the state vector $\mathbf{x}(k)$ after a change in the reference value $\mathbf{x}_{r}(k)$.

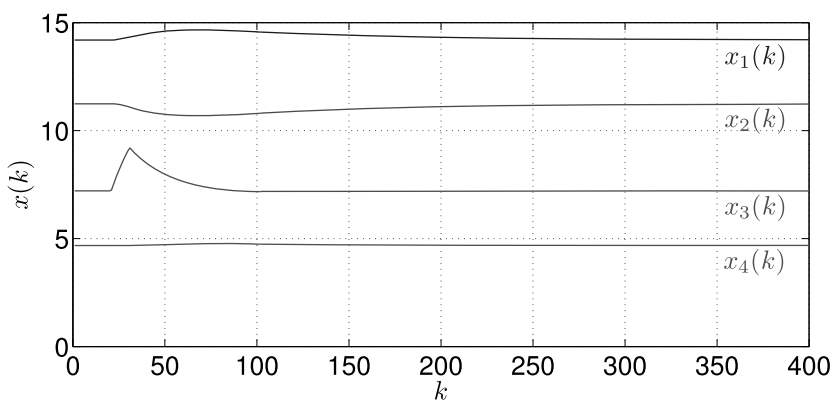

Fig. 6. Stable closed-loop system dynamics perturbed by the external disturbance $d_{1}(k)$.

at $t=10 \mathrm{~s}$ but recovers its stable stationary state $\mathbf{x}_{e}$ about $200 \mathrm{~s}$ later. The switching surface $\mathbf{s}(k)$, the vector error $\mathbf{e}(k)$ and the control law $\mathbf{u}(k)$ dynamics are shown in Figs. 759 respectively.

The second robustness test consists in adding Gaussian noise $\eta(k)$ to the input $u_{1}(k)$, where the mean and variance of $\eta(k)$ are $E(\eta(k))=10$ and $\sigma(\eta(k))=$ 10, respectively (see Fig. 10). Despite this perturbation, the controller stabilizes the system around the stationary point $\mathbf{x}(0)$, as can be seen in Fig. 11

Nonideal sliding mode considerations. To explain how the design parameter $\mathbf{E}$ affects the nonideal sliding mode, let us chose another value for $\mathbf{E}$ such that

$$
\mathbf{E}=\mathbf{E}_{2}=\left[\begin{array}{rr}
4 & -3 \\
-3 & 4
\end{array}\right]
$$

For the state vector feedback gain matrix $\mathbf{K}$ given in (57), the following switching function is obtained with 16):

$$
\mathbf{C}_{2}=\left[\begin{array}{rrrr}
12.204 & 86.811 & -319.526 & 380.667 \\
56.656 & -23.240 & 390.337 & -313.438
\end{array}\right] .
$$

As expected, the system matrix $\hat{\mathbf{\Phi}}_{S M, 2}$ for the ideal SM for $\mathbf{C}=\mathbf{C}_{2}$ coincides with the one given in 60 . For the simulations, the control law given in 61 is chosen, where 


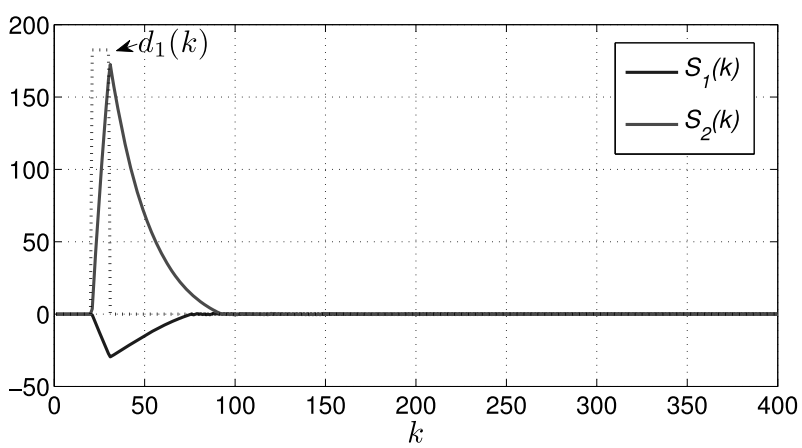

Fig. 7. Surface dynamics for the closed loop system simulated with the external disturbance $d_{1}(k)$

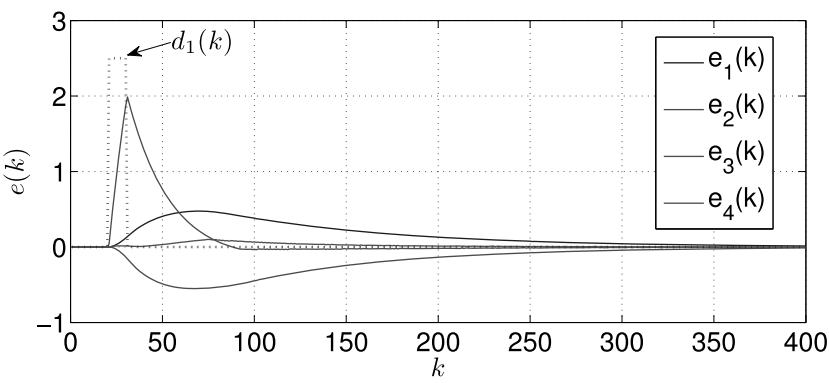

Fig. 8. Error dynamics $\mathbf{e}(k)$ for the pulse disturbance $d_{1}(k)$.

$\mathbf{u}(k)$ is disturbed by the signal noise $\eta(k)$ previously defined. The control law is designed to guarantee that $\mathbf{s}(k)=0$, i.e., $\mathbf{e}(k)=0$ and, as can be seen in Fig. 12, it is achieved for both specifications matrix of $\mathbf{E}$. However, since $\mathbf{E}_{1} \neq \mathbf{E}_{2}$, the control law effort differs considerably in each case, as can be seen in Fig. 13. Hence, it is clear that $\mathbf{E}$ can be used for improving the controlled system performance during the sliding mode phase.

This numerical simulation shows that a switching surface specified as in (16) yields asymptotically stable nonideal sliding mode dynamics for nonlinear discrete time MIMO systems whose dynamics are given in the affine variable state representation. In addition, it is illustrated how the definition of the parameter $\mathbf{E}$ affects the nonideal controlled system behaviour.

\section{Conclusion and future works}

This research work proposed a simple scheme for designing switching surfaces applied to a discrete time affine nonlinear MIMO system. The presented methodology makes use of linearized systems dynamics. We integrated the linearizing procedure with the surface scheme in a unique equation which leads to a novel and powerful design approach. Due to using the well-known control methodologies applied to linear systems, such as pole placement or LQR, the researcher can apply all of

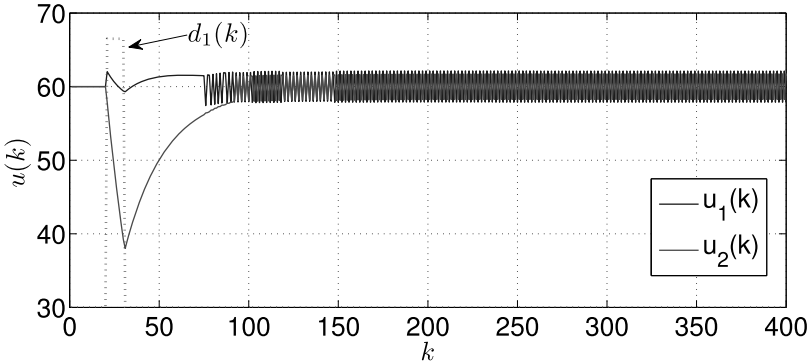

Fig. 9. Control law $\mathbf{u}(k)$ for perturbed closed loop systems
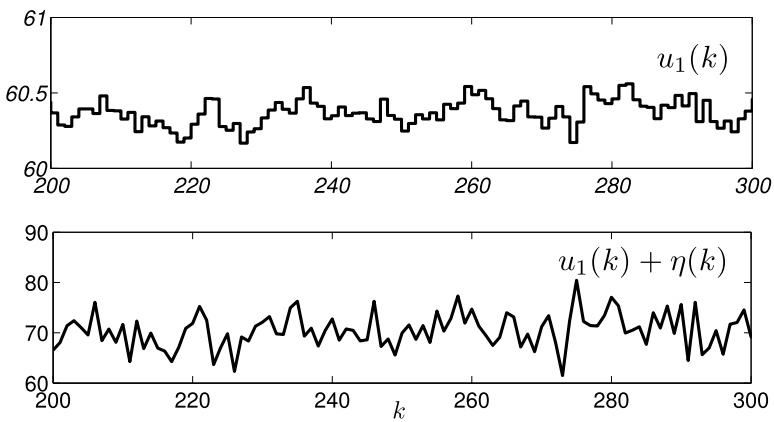

Fig. 10. Nominal control law $u_{1}(k)$ and the perturbed control law $\eta(k)+u_{1}(k)$.

these different design criteria to a nonlinear DVSC control problem.

The absence of any coordinate transformation eliminates the hard task of obtaining those, which in turn reduces the design effort. In addition, the proposed method is the only one that allows designers to adjust both the reaching and nonideal sliding modes through the matrix $\mathbf{E}$ for nonlinear MIMO systems. Moreover, future research works that analyze the pseudo-sliding dynamics for a controlled system with the proposed switching surface scheme can be conducted in order to obtain new reaching law conditions or improved control laws. For all these reasons, this research work can be considered a useful tool for the design of sliding surfaces for controlling nonlinear MIMO systems via DVSC. The design rules presented for the design of DVSC according to the proposed methodology constitute an easy brief guideline for designers.

On the other hand, the proposed methodology makes use of the Jacobian or Taylor linearizing criteria. Since other optimal linearization approaches applied to nonlinear dynamical systems have been proposed (see Ababneh et al., 2011), these linearization methods can be used instead of the Jacobian criterion to verify if some performance features of the controlled system dynamics are improved. 


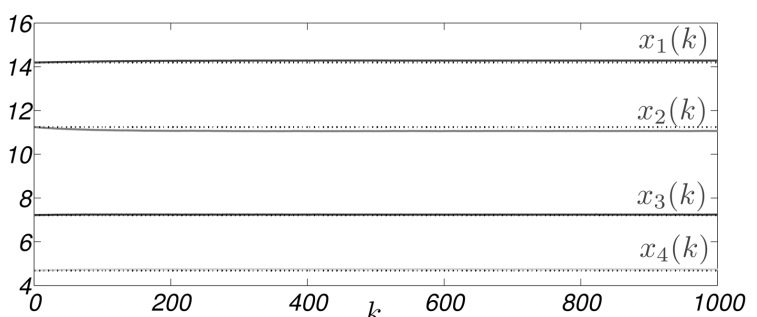

Fig. 11. State vector dynamics $x(k)$ for the disturbance $d_{1}(k)$.
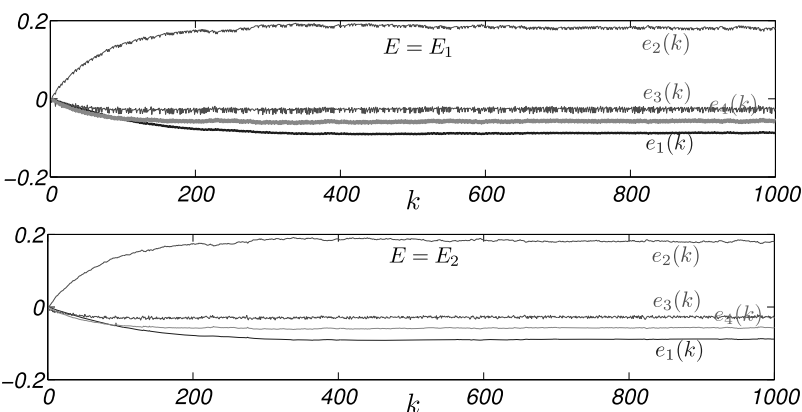

Fig. 12. Comparisons between the tracking error for $\mathbf{E}=\mathbf{E}_{1}$ and $\mathbf{E}=\mathbf{E}_{2}$.

\section{Acknowledgment}

This work has been funded by the Spanish Ministry of Economy and Competitiveness (Assisted Navigation Through Natural Language (NAVEGASE) project no. DPI2014-53525-C3-1-R).

\section{References}

Ababneh, M., Salah, M. and Alwidyanm, K. (2011). Linearization of nonlinear dynamical systems: A comparative study, Jordan Journal of Mechanical and Industrial Engineering 5(6): 567-571.

Ackermann, J. and Utkin, V. (1994). Sliding mode control design based on Ackermann's formula, Proceedings of the 33rd IEEE Conference on Decision and Control, Lake Buena Vista, FL, USA, Vol. 4, pp. 3622-3627.

Åström, K.J. and Wittenmark, B. (1997). Computer-Controlled Systems. Theory and Design, 3rd Edn., Prentice-Hall, Upper Saddle River, NJ.

Bartoszewicz, A. (1998). Discrete-time quasi-sliding-mode control strategies, IEEE Transactions on Industrial Electronics 45(4): 633-637.

Bartoszewicz, A. and Leśniewski, P. (2014). An optimal sliding mode congestion controller for connection-oriented communication networks with lossy links, International Journal of Applied Mathematics and Computer Science 24(1): 87-97, DOI: 10.2478/amcs-2014-0007.

Camacho, O. and Smith, C.A. (2000). Sliding mode control: An approach to regulate nonlinear chemical processes, ISA Transactions 39(2): 205-218.
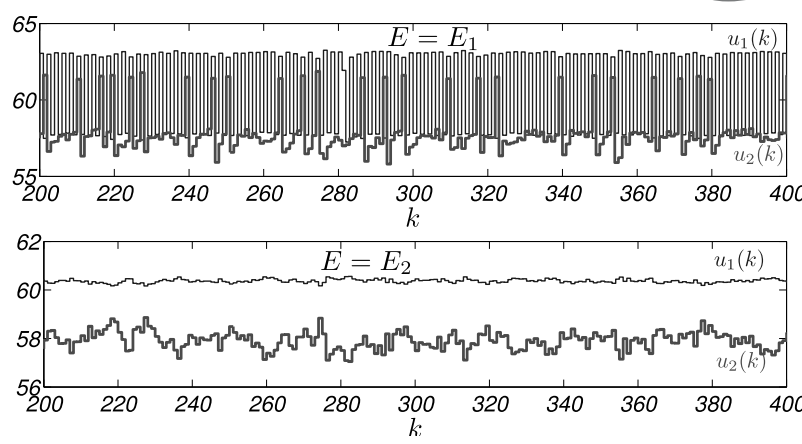

Fig. 13. Comparisons between the control law for $\mathbf{E}=\mathbf{E}_{1}$ and $\mathbf{E}=\mathbf{E}_{2}$.

Choi, H.H. (2003). An LMI-based switching surface design method for a class of mismatched uncertain systems, IEEE Transactions on Automatic Control 48(9): 1634-1638.

DeCarlo, R., Zak, S. and Matthews, G. (1988). Variable structure control of nonlinear multivariable systems: A tutorial, Proceedings of the IEEE 76(3): 212-232.

Dorling, C. and Zinober, A. (1986). Two approaches to hyperplane design in multivariable structure control systems, International Journal of Control 44(1): 65-82.

Draženović, B., Milosavljević, Č. and Veselić, B. (2013). Comprehensive approach to sliding mode design and analysis in linear systems, in B. Bandyopdhyay et al. (Eds.), Advances in Sliding Mode Control: Concept, Theory and Implementation, Springer, Heidelberg, pp. 1-19.

Edwards, C. and Spurgeon, S. (2003). Linear matrix inequality methods for designing sliding mode output feedback controllers, IEE Proceedings: Control Theory and Applications 150(5): 539-545.

Furuta, K. (1990). Sliding mode control of a discrete system, Systems and Control Letters 14(2): 145-152.

Furuta, K. and Pan, Y. (1995). A new approach to design a sliding sector for VSS controller, Proceedings of the 1995 American Control Conference, Seatle, WA, USA, Vol. 2, pp. 1304-1308.

Furuta, K. and Pan, Y. (2002). Discrete-time variable structure control, in X. Yu and J.-X. Xu (Eds.), Variable Structure Systems: Towards the 21st Century, Springer, Berlin, pp. 57-81.

Gao, W. and Hung, J. (1993). Variable structure control of nonlinear systems: A new approach, IEEE Transactions on Industrial Electronics 40(1): 45-55.

Gao, W., Wang, Y. and Homaifa, A. (1995). Discrete-time variable structure control systems, IEEE Transactions on Industrial Electronics 42(2): 117-122.

Gatzke, E.P., Meadows, E.S., Wang, C. and Doyle, F.J. (2000). Model based control of a four-tank system, Computers \& Chemical Engineering 24(2): 1503-1509.

Ghaffari, A. and Yazdanpanah, M. (2008). Nonlinear sliding surfaces: Computing and existence of solution, International Conference on Control, Automation and Systems, ICCAS 2008, Seoul, Korea, pp. 1610-1615. 
Hung, J.Y., Gao, W. and Hung, J.C. (1993). Variable structure control: A survey, IEEE Transactions on Industrial Electronics 40(1): 2-22.

Johansson, K. and Nunes, J. (1998). A multivariable laboratory process with an adjustable zero, Proceedings of the 1998 American Control Conference, Philadelphia, PA, USA, Vol. 4, pp. 2045-2049.

Kim, K.-S., Park, Y. and Oh, S.-H. (2000). Designing robust sliding hyperplanes for parametric uncertain systems: A Riccati approach, Automatica 36(7): 1041-1048.

Lin, Y., Shi, Y. and Burton, R. (2013). Modeling and robust discrete-time sliding-mode control design for a fluid power electrohydraulic actuator (EHA) system, IEEE/ASME Transactions on Mechatronics 18(1): 1-10.

Milosavljević, Č. (1985). General conditions for the existence of a quasi-sliding mode on the switching hyperplane in discrete variable structure systems, Automation and Remote Control 46(3): 307-314.

Mondal, S., Gokul, T. and Mahanta, C. (2012). Chattering free sliding mode controller for mismatched uncertain system, 7th IEEE International Conference on Industrial and Information Systems (ICIIS), Chennal, India, pp. 1-6.

Nadzinski, G., Vladev, G. and Zheng, Y. (2012). A design of discrete-time SMC for nonlinear systems based on fuzzy T-S model, 6th IEEE International Conference on Intelligent Systems (IS), Sofia, Bulgaria, pp. 317-324.

Pai, M.-C. (2008). Discrete-time output feedback sliding mode control for uncertain systems, Journal of Marine Science and Technology 16(4): 295-300.

Perruquetti, W. and Barbot, J.-P. (2002). Sliding Mode Control in Engineering, Marcel Dekker, New York, NY.

Potts, R. and Yu, X. (1991). Discrete variable structure system with pseudo-sliding mode, Journal of the Australian Mathematical Society B: Applied Mathematics 32(04): 365-376.

Qu, S., Xia, X. and Zhang, J. (2014). Dynamics of discrete-time sliding-mode-control uncertain systems with a disturbance compensator, IEEE Transactions on Industrial Electronics 61(7): 3502-3510.

Rui, D. and Dong-wei, S. (2011). Optimal sliding mode design for nonlinear discrete-time systems, 30th Chinese Control Conference (CCC), Yantai, China, pp. 738-742.

Sira-Ramírez, H. (1986). Variable structure control of nonlinear systems through simplified uncertain models, 25th IEEE Conference on Decision and Control, Athens, Greece, pp. 2037-2041.
Sira-Ramírez, H. (1991). Non-linear discrete variable structure systems in quasi-sliding mode, International Journal of Control 54(5): 1171-1187.

Soroush, M. and Kravaris, C. (1992). Discrete-time nonlinear controller synthesis by input/output linearization, AIChE Journal 38(12): 1923-1945.

Spurgeon, S. and Davies, R. (1993). A nonlinear design approach for sliding mode control systems, Proceedings of the 32nd IEEE Conference on Decision and Control, San Antonio, TX, USA, Vol. 2, pp. 1440-1445.

Spurgeon, S. and Pugh, A. (1991). On output deadbeat control of discrete-time multivariable systems, IEEE Transactions on Automatic Control 36(7): 894-896.

Su, W.C., Drakunov, S.V. and Özgüner, Ü. (1994). Constructing discontinuity planes for variable structure systems-A Lyapunov approach, American Control Conference, Baltimore, MD, USA, Vol. 1, pp. 1169-1173.

Su, W.-C., Drakunov, S.V. and Özgüner, Ü. (1996). Constructing discontinuity surfaces for variable structure systems: A Lyapunov approach, Automatica 32(6): 925-928.

Tapia, A., Márquez, R., Bernal, M. and Cortez, J. (2014). Sliding subspace design based on linear matrix inequalities, Kybernetika 50(3): 436-449.

Utkin, V. (1977). Variable structure systems with sliding modes, IEEE Transactions on Automatic Control 22(2): 212-222.

Utkin, V., Guldner, J. and Shi, J. (1999). Sliding Mode Control in Electro-mechanical Systems, CRC Press, Boca Raton, FL.

Utkin, V. and Yang, K. (1978). Methods for construction of discontinuity planes in multidimensional variable structure systems, Automation and Remote Control 39(6): 1466-1470.

Wang, Y., Xia, Z., Jiang, Z. and Xie, G. (2011). A quasi-sliding mode variable structure control algorithm for discrete-time and time-delay systems, Chinese Control and Decision Conference, Mianyang, China, pp. 107-110.

Yadav, N. K. and Singh, R. (2012). Robust discrete-time nonlinear sliding mode controller with plant uncertainties, International Journal of Engineering, Science and Technology 4(1): 38-45.

Yu, S., Yu, X. and Qian, W. (2000). Time delayed discrete variable structure control with quasi-sliding modes, in $\mathrm{X}$. $\mathrm{Yu}$ and J.-X. Xu (Eds.), Advances in Variable Structure Systems: Analysis, Integration and Applications, World Scientific, Singapore, pp. 84-92.

Zhang, X., Wang, P., Yan, M. and Ju, Y. (2010). Discrete-time sliding mode control of nonlinear time-delay systems based on T-S fuzzy model, International Conference on Intelligent Control and Information Processing (ICICIP), Dalian, China, pp. 304-309. 


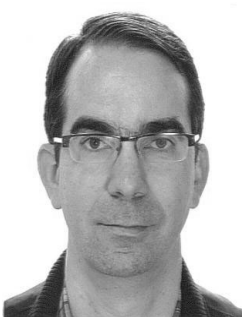

José Darío Luis-Delgado (BSc and MSc in electronic engineering) received the $\mathrm{PhD}$ degree in automation, robotics and industrial electronics in 2016 from Universidad Politécnica de Madrid (Spain) with a thesis on analysis and design of multivariable discrete time slide-mode control systems. He has been working in industry related to automation, telecommunications and electronics for more than 20 years and is currently the chief of the Automation and Control Department of Canal de Comunicaciones Unidas (Madrid, Spain), whose main activity is focused on the automation of water cycle industrial processes. $\mathrm{He}$ has taught as an associate professor at several universities (electronics, automation and control), and has worked as a researcher in artificial vision and robotics at Universidad de Murcia (Spain) and in discrete time signal processing at Universidad Simn Bolvar (Venezuela).

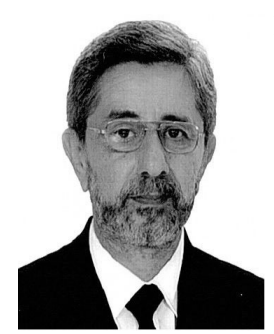

Basil Mohammed Al-Hadithi (BSc in control and system engineering, MSc in control and instrumentation engineering) received the $\mathrm{PhD}$ degree in process control and artificial intelligence in 2002 from Universidad Politécnica de Madrid (UPM) (Spain) with a thesis on analysis, design and stability of fuzzy slide-mode control systems. $\mathrm{He}$ is an associate professor at UPM. His teaching activity covers control engineering and analogue electronics. He is a researcher at the Centre for Automation and Robotics UPM-CSIC. His interest is focused mainly on fuzzy control and slide-mode control. He is a co-editor the book entitled Robot Manipulators, Trends and Development (Intech, 2010). Moreover, he has participated in several research projects and industrial contracts with companies. He is a board member and a reviewer of several international scientific societies.

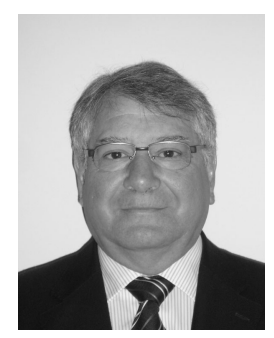

Agustín Jiménez is a professor at the Department of Automatic Control, Industrial Electronics and Computer Science of the Technical University of Madrid. Born in Toledo in 1952, he obtained his engineering degree in 1974 and his $\mathrm{PhD}$ in 1978. His main teaching and research activities have been related to the development and application of advanced technologies for process control, especially the integration of artificial intelligence technologies with conventional control applications. His experience in R\&D projects is very wide, including numerous international projects, especially those financed by the European Community, the Spanish National Plan and the ones regarding technological transfer to industry.

\section{Appendix}

\section{A1. First order Taylor expansion term linearization method}

Given $f: \mathbb{R} \rightarrow \mathbb{R}$ such that $f(x)$ is infinitely many times differentiable at some complex or real point $x_{e}, f(\cdot)$ can be approximated around $x_{e}$ through the following Taylor expansion:

$$
f(x)=\sum_{i=0}^{\infty} \frac{f^{(i)}\left(x_{e}\right)}{i !}\left(x-x_{e}\right)^{i},
$$

where $f^{(i)}$ denotes the $i$-th derivative of $f$. For the first term of A1, we have

$$
f(x) \approx f\left(x_{e}\right)+f^{\prime}\left(x_{e}\right)\left(x-x_{e}\right) .
$$

For a general dynamic system

$$
\dot{x}(t)=f(x),
$$

assume that for $x=x_{e}$ we have $f\left(x_{e}\right)=0$. For the case of $x_{e}=0$, we have

$$
\dot{x}(t) \approx f^{\prime}(0)\left(x(t)-x_{e}\right) .
$$

For the general multivariable case $\mathbf{f}: \mathbb{R}^{n} \rightarrow \mathbb{R}^{n}$, i.e., for f $(\cdot)$ defined as

$$
\mathbf{f}(x)=\left[\begin{array}{c}
f_{1}(x) \\
f_{2}(x) \\
\vdots \\
f_{2}(x)
\end{array}\right]
$$

where $x(t)=\left[\begin{array}{llll}x_{1}(t) & x_{1}(t) & \cdots & x_{n}(t)\end{array}\right]$, we have

$$
\dot{x}(t) \approx D f\left(x_{e}\right)\left(x(t)-x_{e}\right)
$$

where $D \mathbf{f}\left(x_{e}\right)$ is the Jacobian matrix defined as

$$
D \mathbf{f}(x)=\mathbf{J}=\left[\begin{array}{cccc}
\frac{\partial f_{1}}{\partial x_{1}} & \frac{\partial f_{1}}{\partial x_{2}} & \cdots & \frac{\partial f_{1}}{\partial x_{n}} \\
\frac{\partial f_{2}}{\partial x_{1}} & \frac{\partial f_{2}}{\partial x_{2}} & \cdots & \frac{\partial f_{2}}{\partial x_{n}} \\
\vdots & \vdots & \ddots & \vdots \\
\frac{\partial f_{n}}{\partial x_{1}} & \frac{\partial f_{n}}{\partial x_{2}} & \cdots & \frac{\partial f_{n}}{\partial x_{n}}
\end{array}\right]
$$

\section{A2. Forward Euler method}

The well-known forward Euler method, or simply the Euler method, is an explicit numerical approximation methodology for numerically solving ordinary differential equations, which produces first-order solutions. For the differential equation

$$
\dot{x}(t)=f(x, t)
$$

a numerical approximation of $x(t)$ at $t=k_{0}+k T$, where $k \in \mathbb{Z}$ is an integer time step with temporal separation of $T$, can be made using

$$
x(k+1)=x(k)+T f(k, x(k)) .
$$

It is known that the global approximation error is proportional to the step size $T$, and therefore the global error decreases as $T \rightarrow 0$.

Received: 14 October 2015 Revised: 30 January 2016 Re-revised: 25 August 2016 Accepted: 31 October 2016 